Les avancées en génétique et en médecine personnalisée, comprenant aussi en partie la médecine de précision, sont utilisées au quotidien dans le domaine de la santé. L'enthousiasme régnant dans ce domaine devrait, de la même façon que la révolution numérique, être reçu avec un scepticisme réfléchi. En fin de compte, la condition humaine ne doit pas être oubliée: la médecine personnalisée étant bien plus vaste qu'une vision sur les molécules et les gènes. Elle se base en effet sur l'empathie et les relations avec les patients. Prendre part à la conférence sur ce thème en vaut la peine; il y sera entre autres question des défis que représente la liaison entre la médecine de précision au niveau nano- et microscopique et la complexité individuelle aux niveaux méso et macro.

\title{
La santé personnalisée du point de vue de la santé publique
}

\section{Julia Dratva \\ Dr méd., MD MPH, Présidente de la Société suisse des médecins spécialistes en prévention et santé publique}

Jamais auparavant nous n'avons disposé d'autant de données provenant de différentes sources. De nouveaux horizons s'ouvrent ainsi pour les sciences de la santé. Le dépouillement de données génétiques et biochimiques ainsi que les informations sur l'activité physique et le mode de vie permettent de développer de manière encore plus ciblée des diagnostics et thérapies pour certains sous-groupes de personnes afin de proposer, d'une part, une prévention et des traitements personnalisés et, d'autre part, prendre des mesures de prévention efficaces pour la population. Ces informations personnalisées peuvent être comparées à d'autres, p. ex. sur les polluants atmosphériques ou le climat, et peuvent apporter de nouvelles connaissances importantes. Des chercheurs suisses ont par exemple prouvé l'existence d'un lien entre la pollution de l'air au lieu de domicile et le risque de diabète. La condition à cela est l'harmonisation de l'accès aux données et une compréhension commune de la santé de la population.

La Conférence Suisse de Santé Publique 2017 sera l'occasion d'aborder ces sujets. Nous discuterons dans quelle mesure la médecine personnalisée est compatible avec la santé publique et quelles sont les nouvelles possibilités qu'elle présente pour la prévention et la santé publique. Il faudra également tenir compte des aspects éthiques, par exemple en ce qui concerne la protection des données. Enfin, nous mettrons en lumière le marché de la santé personnalisé en plein essor et ses conséquences ainsi que la question de l'accès équitable à la médecine personnalisée. Nous avons concocté un programme varié et orienté vers la pratique qui pourra être source de suggestions et d'idées pour des coopérations. Nous nous réjouissons d'avance de vous accueillir à Bâle à l'occasion de la Conférence Suisse de Santé Publique 2017 et de discuter les questions soulevées avec vous ainsi qu'avec des experts et expertes internationaux.

La Conférence Suisse de Santé Publique est organisée par Santé publique Suisse (www.public-health.ch), la Swiss School of Public Health (www.ssphplus.ch) et des organisations partenaires. Chaque année, une institution académique suisse du domaine de la santé publique accueille la conférence et assure la direction scientifique et thématique. L'hôte de la Conférence Suisse de Santé Publique 2017, placée sous le thème «La santé personnalisée du point de vue de la santé publique», est l'Institut tropical et de santé publique suisse (Swiss TPH). Quant à la Société suisse des médecins spécialistes en prévention et santé publique (www. sgpg.ch), elle est responsable de la formation médicale postgraduée et continue dans le domaine de la prévention et de la santé publique en Suisse et s'engage pour de bonnes conditions-cadre en faveur de la santé de la population. Dans ce sens, la SSSP recommande vivement à ses membres et à tous les confrères qui s'intéressent à la santé publique de s'inscrire à la conférence.

http://conference.public-health.ch 